\title{
Reduction of the Multipactor Threshold Due to Electron Cyclotron Resonance
}

\author{
V.E. Semenov, N.A. Zharova, N.I. Zaitsev, A.K. Gvozdev, A.A. Sorokin, M. Lisak ${ }^{1}$, J. \\ Rasch $^{1}$, J. Puech ${ }^{2}$.
}

Institute of Applied Physics, Nizhny Novgorod, Russia

1 Chalmers University of Technology, Göteborg, Sweden

2 Centre National d''Etudes Spatiales, 31401 Toulouse Cedex 9, France

\begin{abstract}
Single surface multipactor on metal surfaces is studied in the case when the microwave electric field is superimposed on a dc electric and a permanent magnetic field. Based on a simple analysis of the electron motion it is predicted that considerable reduction in the multipactor threshold is possible when the electron cyclotron frequency equals the microwave field frequency and the permanent magnetic field has the proper orientation with respect to the metal surface. The prediction is confirmed by numerical simulations, and some experimental indications of the reduced multipactor threshold are also presented.
\end{abstract}




\section{Introduction}

Multipactor discharges occur in high power microwave systems operating close to vacuum conditions. It is caused by secondary electron emission from device walls when the latter are bombarded by energetic electrons. If the secondary electrons are accelerated by the microwave field up to energies that are enough to produce generation of new secondary electrons, the multipactor avalanche grows which results in a number of undesirable effects such as generation of electromagnetic noise, impedance mismatch, extra heating of the system and even hardware damage. Therefore, correct predictions of the multipactor threshold become a necessary prerequisite for the design of many modern microwave applications including high power generators [1], particle accelerators [2], and space borne communications systems [3]. Considerable attention has also been paid to studies of multipactor mitigation using artificial surface coating [4] or by applying a permanent magnetic field to the system [5-10]. On the other hand, it has been stated in a number of papers that similar threshold parameters occur for multipactor in systems with permanent magnetic fields as in systems without such fields [11-16]. In these cases however, the magnetic field was quite weak, and did not affect the electron velocity significantly. But even a weak magnetic field may still have a large effect on the resonance conditions. Situations have also been observed when the presence of a dc magnetic field causes a reduction in the multipactor threshold [10,17-21]. Thus, there is some uncertainty concerning the effect of a magnetic field on the multipactor threshold and some clarification of the situation seems desirable. Such a clarification is given in the present paper based on a detailed analysis of the electron motion which makes it possible to distinguish between different effects determining the multipactor threshold. Main attention is given to a study of the single-surface multipactor on a metal surface. For this particular case, a dangerous combination of parameters is determined that leads to a considerable reduction in the multipactor threshold due to electron cyclotron resonance. This dangerous parameter combination can be met in the output section of some high-power microwave generators where a relatively strong non-uniform magnetic field is superimposed with a relatively weak dc electric field induced by the electron beam. The prediction is confirmed in numerical simulations and some experimental verification is also discussed. 


\section{Peculiarities of electron motion in a uniform electromagnetic field}

The multipactor avalanche grows when secondary electrons strike solid surfaces with sufficiently high impact energy. Therefore it seems appropriate to start an estimate of the multipactor threshold with an analysis of the maximum energy acquired by electrons in a spatially uniform electromagnetic field. The analysis can be carried out using a simplified model within which the action of the microwave magnetic field on the electron motion as well as relativistic effects are neglected. Under this approximation, the electron motion is governed by the equation

$$
m \frac{d^{2} \vec{r}}{d t^{2}}=-e \cdot\left\{\vec{E}_{0}+\vec{E}_{\omega} \cdot \sin (\omega t)+\frac{1}{c} \vec{V} \times \vec{B}\right\},
$$

where $m$ and $-e$ denote electron mass and charge, vectors $\vec{r}$ and $\vec{V}=d \vec{r} / d t$ describe the instantaneous position and velocity of the electron, $c$ denotes the velocity of light in vacuum, $\vec{E}_{0}$ the dc component of the electric field strength, $\vec{E}_{\omega}$ the amplitude of the microwave electric field strength, $\omega$ the angular frequency of this field and $\vec{B}$ the induction of the permanent magnetic field. In the simplest case, when only the microwave field is present (the basic model for the multipactor between two metal plates), the solution of (1) can be presented as a superposition of a free uniform motion with constant velocity, $\vec{V}_{f}$, and oscillations forced by the microwave field:

$$
\vec{V}_{o s c}=\vec{V}_{\omega} \cdot \cos (\omega t), \quad \vec{r}_{o s c}=\left(\vec{V}_{\omega} / \omega\right) \cdot \sin (\omega t),
$$

where $\vec{V}_{\omega}=e \vec{E}_{\omega} / m \omega$. The amplitude, $V_{\omega}$, of the electron velocity oscillations (driven by the microwave field only) will be called the oscillatory velocity. Taking into account initial conditions (corresponding to the moment, $t_{e}$, of electron emission), one can express $\vec{V}_{f}$ via $\vec{V}_{\omega}$ and the electron initial velocity $\vec{V}_{0}$ :

$$
\vec{V}_{f}=\vec{V}_{0}-\vec{V}_{\omega} \cdot \cos \left(\omega t_{e}\right) .
$$

Combining (2) and (3) one finds that the electron velocity cannot exceed the value

$$
V_{\max }=V_{0}+2 V_{\omega} .
$$

The maximum electron velocity (4) is achieved when the proper emission time (which provides the equality $\left.\sin \left(\omega t_{e}\right)=0\right)$ is combined with the proper duration of the electron flight time (which should be equal to an odd number of half microwave cycles). Specifically, these conditions are fulfilled in the case of the so called resonance regime of double-surface 
multipactor between parallel metal plates. The resonance occurs [22, 23] when the gap width, $h$, equals

$$
h=\frac{\pi N}{\omega}\left[V_{0}+V_{\omega} \cos \left(\omega t_{e}\right)\right]+\frac{2}{\omega} V_{\omega} \sin \left(\omega t_{e}\right)
$$

where it is assumed that the initial velocity is normal to the emission surface, and an odd number, $N$, denotes the resonance order. The corresponding impact velocity is given by $V_{i m p}=V_{0}+2 V_{\omega} \cos \left(\omega t_{e}\right)$, which is close to $V_{\max }$ because the emission phase is close to zero $\left(\omega t_{e}<<1\right.$ in case of the stable resonance [23]). Therefore a simple estimate for the lower envelope of the multipactor resonance bands is obtained simply by using the equality

$$
V_{0}+2 V_{\omega}=V_{1}
$$

where the impact energy, $m V_{1}^{2} / 2$, corresponds to the first cross over point of the secondary emission curve.

When the microwave field is superimposed on a permanent magnetic field, the latter does not disturb the electron motion along the direction of the magnetic field, whereas in the transverse plane this motion is changed considerably. In this plane the solution of (1) can be represented as a superposition of rotation with constant velocity, $\left|\vec{V}_{\perp f}\right|$, at the cyclotron frequency, $\omega_{B}=e B /(m c)$ and oscillations, $\vec{V}_{\perp o s c}$, forced by the transverse component of the microwave field. Without any loss in generality we can use a Cartesian coordinate system, where the magnetic field is aligned with the z-axis, and the perpendicular component of the electric field with the x-axis. In this case, using a harmonic electric field, $\vec{E}_{\perp}=\vec{E}_{\perp \omega} \cdot \sin (\omega t)$, and the initial condition, $\vec{V}_{\perp}\left(t_{e}\right)=\vec{V}_{0}$, we find [13]:

$$
\vec{V}_{\perp}=\vec{V}_{\perp o s c}+\vec{V}_{\perp f}
$$

where the vector components are

$$
\begin{gathered}
V_{\perp o s c, x}=V_{\perp \omega} \cos (\omega t) \\
V_{\perp o s c, y}=V_{\perp \omega} \frac{\omega_{B}}{\omega} \sin (\omega t) \\
V_{\perp f, x}=\cos \left(\omega_{B}\left(t-t_{e}\right)\right)\left[V_{x, 0}-V_{\perp \omega} \cos \left(\omega t_{e}\right)\right]-\sin \left(\omega_{B}\left(t-t_{e}\right)\right)\left[V_{y, 0}-V_{\perp \omega} \frac{\omega_{B}}{\omega} \sin \left(\omega t_{e}\right)\right] \\
V_{\perp f, y}=\sin \left(\omega_{B}\left(t-t_{e}\right)\right)\left[V_{x, 0}-V_{\perp \omega} \cos \left(\omega t_{e}\right)\right]+\cos \left(\omega_{B}\left(t-t_{e}\right)\right)\left[V_{y, 0}-V_{\perp \omega} \frac{\omega_{B}}{\omega} \sin \left(\omega t_{e}\right)\right]
\end{gathered}
$$


and $V_{\perp \omega}=\frac{e E_{\perp \omega}}{m \omega} \cdot \frac{\omega^{2}}{\omega^{2}-\omega_{B}^{2}}$. The motion of the electron is composed of the two vectors, $\vec{V}_{\perp o s c}$ and $\vec{V}_{\perp f}$, where the magnitude of the first vector is independent of the emission phase, and is varying in time. It is given by

$$
\left|\vec{V}_{\perp o s c}\right|^{2}=V_{\perp o s c}^{2}=V_{\perp o s c, x}^{2}+V_{\perp o s c, y}^{2}=V_{\perp \omega}^{2}\left[1-\sin ^{2}(\omega t)\left(1-\frac{\omega_{B}^{2}}{\omega^{2}}\right)\right]
$$

whereas the second vector has a magnitude

$$
V_{\perp f}{ }^{2}=V_{0}^{2}+V_{\perp \omega}^{2} \cos ^{2}\left(\omega t_{e}\right)+V_{\perp \omega}^{2} \frac{\omega_{B}^{2}}{\omega^{2}} \sin ^{2}\left(\omega t_{e}\right)-2 V_{x, 0} V_{\perp \omega} \cos \left(\omega t_{e}\right)-2 V_{y, 0} V_{\perp \omega} \frac{\omega_{B}}{\omega} \sin \left(\omega t_{e}\right)
$$

which is constant in time, and determined by the emission phase and velocity. Thus, the maximum velocity that can be achieved is determined by $V_{\perp f}$, and its dependence on the emission phase. In the limit where $V_{0}<<V_{\perp \omega}$, there are two possible maxima, with positions determined by the relative strength of the magnetic field. The maxima are located at $\cos \left(\omega t_{e}\right)=0$, if $\omega_{B} / \omega>1$, and $\sin \left(\omega t_{e}\right)=0$, if $\omega_{B} / \omega<1$. When $\omega_{B} / \omega>1$, the magnitude of $\vec{V}_{\perp f}$ cannot exceed the value

$$
V_{\perp f, \max }=\sqrt{V_{0}^{2}+2 V_{y, 0} V_{\perp \omega} \frac{\omega_{B}}{\omega}+V_{\perp \omega}^{2} \frac{\omega_{B}^{2}}{\omega^{2}}} \leq V_{\perp \omega} \frac{\omega_{B}}{\omega}+V_{0}
$$

which is completely maximized when the emission velocity is entirely in the y-direction, i.e. $V_{0}=V_{y, 0}$. Consequently, the maximum velocity attainable is achieved when the two vectors are pointing in the same direction, and $\cos (\omega t)=0$. It has a magnitude

$$
V_{\perp \max }=V_{0}+2 V_{\perp \omega} \frac{\omega_{B}}{\omega}
$$

In the same way, the maximum velocity under the condition $\omega_{B} / \omega<1$ is given by

$$
V_{\perp \max }=V_{0}+2 V_{\perp \omega}
$$

The maximum velocities occurs when the velocity vectors are pointing in the same direction (see Fig. 1). This requires that the proper emission time is combined with the proper duration of the electron flight, $\Delta t=t-t_{e}$, which must satisfy the two equalities

$$
\omega \cdot \Delta t=\pi n,\left|\omega-\omega_{B}\right| \cdot \Delta t=\pi(2 k-1),
$$

where $n$ and $k$ are positive integer numbers. The motivation for these conditions are easily found from Fig. 1, since the oscillatory velocity is maximized when the blue arrow is pointing 
either in the negative or positive $\mathrm{x}$-direction, i.e. $\omega \Delta t=\pi n$. And the total velocity (black arrow) is maximized when the red arrow (drift velocity) points in the same direction as the blue one, i.e. $\left|\omega-\omega_{B}\right| \Delta t=\pi(2 k-1)$. As an example one can refer to the resonant regime of multipactor on a single metal surface in crossed microwave electric and permanent magnetic fields [11-16]. Within this resonance, equalities (17) are fulfilled with $n=2 k$.
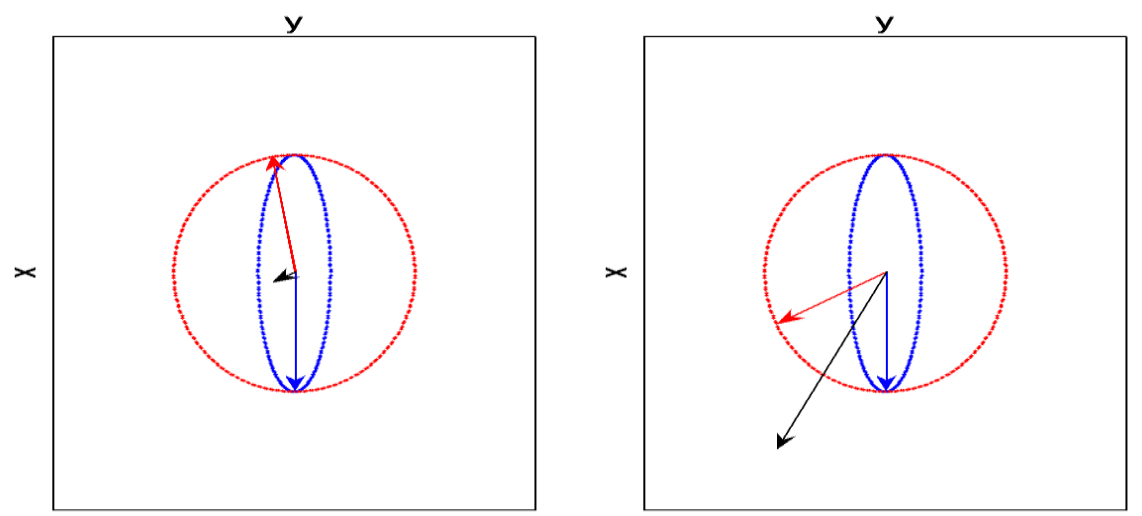

Fig. 1. The relative direction of the electron velocity vectors in the plane perpendicular to the magnetic field. The blue arrow represents $\vec{V}_{\perp s s c}$, the red arrow $\vec{V}_{\perp f}$, and the black arrow the total velocity, $\vec{V}_{\perp}$. The left panel shows the vectors at the time of emission, when the total velocity is small and equal to $\vec{V}_{0}$. At this time the angle between the vectors is very close to $\pi$. The right panel shows the vectors at the time of impact, when $\omega_{B} \Delta t<\pi$. If the electrons are able to move in complete resonance, at the time of impact, both vectors will point in the same direction, in the negative or positive x-direction. Since the blue arrow is rotating with the frequency $\omega$, and the red one with the frequency $\omega_{B}$, direct inspection of the figures leads to the conditions for resonance and maximum impact velocity, (17).

\section{Effect of permanent magnetic field on the multipactor threshold}

According to (16) the maximum electron velocity is not changed significantly in the case of a weak magnetic field (i.e. for $\left.\omega_{B}<<\omega\right)$. However, even a weak magnetic field can affect both equalities (17), especially when the electron flight time significantly exceeds the microwave period. Generally, only the first equality in (17) is responsible for the multipactor resonance (double-surface if $n$ is an odd number or single-surface if $n$ is an even number). A 
variation of the magnetic field strength can either destroy or restore these resonances ${ }^{1}$. Therefore an increase in the magnetic field strength (all other parameters being kept constant) is accompanied by the appearance of local maxima and minima of the multipactor threshold [10]. On the other hand, when the multipactor resonance of fixed order, $n$, is kept (for example, when a variation of the magnetic field is accompanied by a variation of the dc electric field and/or microwave frequency so as to keep the equality $\omega \cdot \Delta t=\pi \cdot n$ ) the value $\omega_{B} \cdot \Delta t$ increases monotonously with increasing magnetic field strength, and the second equality in (17) can become violated. This is not the case for the conventional single-surface multipactor resonance when the dc electric field is absent, the permanent magnetic field is parallel to the emission surface, $\omega \cdot \Delta t=\pi \cdot n, \omega_{B} \cdot \Delta t=\pi$ [12], and both equalities (17) are fulfilled. However, in the case when the dc electric field contributes to the electron return to the surface of emission, the second equality in (17) is violated under the conditions of singlesurface resonance, since the value $\omega_{B} \cdot \Delta t<\pi[12,14]$. This equality is also violated under the conditions of double-surface multipactor resonance where $\omega_{B} \cdot \Delta t<\pi / 2[5,14]$. As a result the velocity vectors have an angular separation of $\pi-\omega \Delta t+\omega_{B} \Delta t$, and their superposition yields an electron impact velocity that is less than $V_{\perp \max }$ :

$$
V_{i m p} \leq \sqrt{V_{\perp \omega}^{2}+\left(V_{0}+V_{\perp \omega}\right)^{2}-2 V_{\perp \omega}\left(V_{0}+V_{\perp \omega}\right) \cdot \cos (\omega \cdot \Delta t) \cdot \cos \left(\omega_{B} \cdot \Delta t\right)} \leq V_{0}+2 V_{\perp \omega} .
$$

Consequently, in these cases the magnetic field can cause some increase in the multipactor threshold even under resonance conditions.

The qualitative difference between single-surface and double-surface multipactor resonances is related to the shape of the electron trajectory. This trajectory forms a closed loop in the case of the double-surface resonance whereas it is close to a semi-circle in the case of the single-surface resonance (see Fig. 2). Therefore in the latter case, a sequence of electron flights is accompanied by a permanent drift perpendicular to both the microwave electric and the permanent magnetic fields. This is not an important effect in the case of infinite parallel plates, but inside a rectangular waveguide, the drift makes the electrons approach the sidewall where the microwave field is zero, which may lead to multipactor suppression [5-7]. It should be noted that inside a coaxial line, a similar drift results in

\footnotetext{
${ }^{1}$ It should be noted that the single-surface multipactor resonance on a metal surface becomes possible even without a permanent magnetic field if e.g. the electron return to the surface of emission is caused by the action of a dc electric field [24].
} 
additional electron rotation around the axis but does not lead to multipactor suppression because of the axial symmetry [10].

a

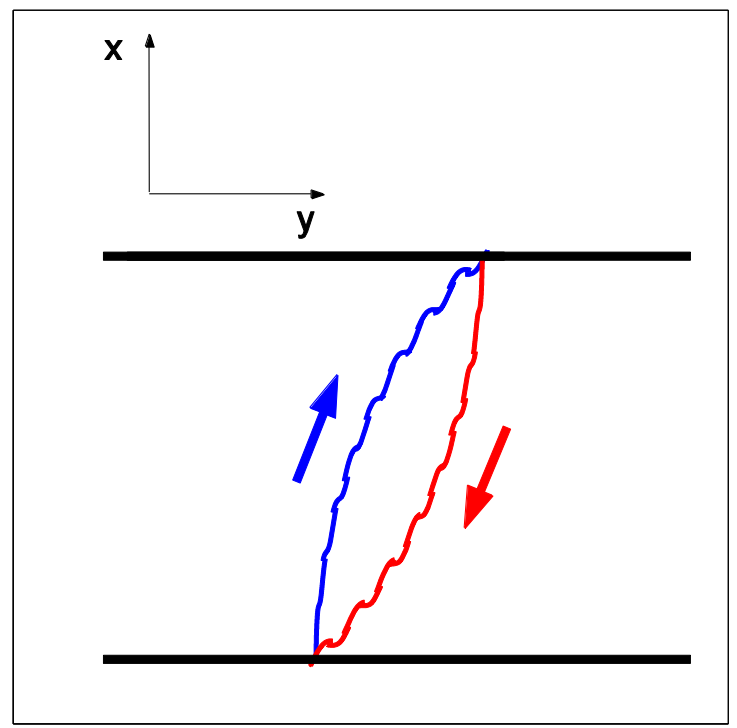

b



Fig. 2. Electron trajectories in the case of double-surface (to the left, $\omega_{B} h /\left(V_{0}+V_{\omega}\right) \cong 0.755$ ) and single-surface (to the right, $\omega_{B} h /\left(V_{0}+V_{\omega}\right) \cong 1.39$ ) multipactor resonance. In both cases, a permanent magnetic field is directed along the $\mathrm{z}$ axis and the microwave electric field is directed along the x-axis, $V_{0}=0.3 \cdot V_{\omega}, \omega h /\left(V_{0}+V_{\omega}\right)=15 \cdot \pi$ ( $h$ denotes gap width between the plates).

When the electron cyclotron frequency is close to the microwave frequency $\left(\omega_{B} \approx \omega\right)$, the permanent magnetic field can affect not only the electron flight time but also lead to a strong increase in the value of $V_{\perp \omega}$ due to electron cyclotron resonance (ECR). The effects of a strong magnetic field have been considered mainly in applications involving the singlesurface multipactor on a dielectric plate [17-20]. The first observation of such multipactor under the condition of $\omega_{B} \approx \omega$ was reported in papers [17, 18] where the permanent magnetic field was parallel both to the microwave electric field and the dielectric surface. In this case the magnetic field does not influence the electron acceleration along the microwave field $\left(\vec{V}_{\perp \omega}=0\right)$. Nevertheless, a noticeable reduction of the multipactor threshold was found at the ECR condition $\left(\omega_{B}=\omega\right)$. The effect was explained by the fact that the equality $\omega \cdot \Delta t=\pi$ results in a maximum of the electron impact velocity given in (4). 
More extended studies on the effect of a permanent magnetic field on the multipactor on a dielectric plate was undertaken in papers $[19,20]$ where different orientations of the magnetic field with respect to the dielectric surface and the polarization of the microwave field were considered. Specifically, it was found that under the ECR condition, the multipactor threshold (in terms of the microwave field amplitude) can be significantly reduced if the permanent magnetic field is perpendicular to the microwave electric field and the electron flight time is long enough to satisfy the second equality in $(17)^{2}$. The latter is possible e.g. when the permanent magnetic field is normal to the dielectric surface and the electron return to the surface is caused by a relatively weak dc electric field which is also normal to the surface. In this case, the electron flight time increases and correspondingly the multipactor threshold (under the ECR condition) decreases with decreasing dc electric field strength.

Reduction of the multipactor threshold under the ECR condition was observed in experiments in a microwave cavity [21]. Within this experiment, the microwave field was parallel to the surface of emission and the permanent magnetic field was perpendicular to this surface, a situation which is similar to the case previously considered in [19, 20]. However, in contrast to the case of multipactor on dielectric surfaces, the microwave field amplitude was zero at the cavity wall and the dc electric field was zero everywhere inside the cavity. Therefore the multipactor was caused by electrons which first drift along the magnetic field away from the cavity wall, then acquire energy from the microwave field under the ECR condition, and then returned back under the action of the ponderomotive (Miller) force [25] caused by the spatial inhomogeneity of the microwave field.

It should be noted that the ECR effect was overlooked in the majority of previous studies of the single-surface multipactor on a metal surface [11, 12, 14], which were concentrated on the case when the permanent magnetic field is parallel to the metal surface, the microwave electric field is uniform and perpendicular to the surface, and the dc electric field pushes emitted electrons back to the surface. In such a case, any emitted electron returns back faster than in a half period of the cyclotron rotation $\Delta t<\pi / \omega_{B}$ and thereby the singlesurface multipactor resonance becomes impossible when $\omega_{B}>\omega / 2$. Based on this fact it was concluded that the single-surface multipactor on a metal surface is completely suppressed by a sufficiently strong magnetic field (with $\omega_{B}>\omega / 2$ ) parallel to the surface. More extended

\footnotetext{
${ }^{2}$ Taking into account the results (6)-(10) of the general analysis of the electron motion, one comes to the conclusion that the electron velocity is not sensitive to the first equality in (16) under the ECR condition (when $\left.\omega_{B} \cong \omega\right)$.
} 
analysis has shown that the single-surface multipactor resonance can occur even in a strong magnetic field parallel to the surface if the direction of the dc electric field is reversed [15]. However, under the multipactor resonant condition $(\omega \cdot \Delta t=2 \pi n)$, the ECR effect is not pronounced since the second equality in (16) is violated at $\omega_{B}=\omega$. Therefore a significant reduction of the multipactor threshold was not found.

A detailed study of the dependence of single-surface multipactor resonance on the magnetic field orientation was completed in [13]. It was found that an increase in the angle, $\theta_{B}$, (up to 45 degree) between the magnetic field line and the metal surface was accompanied by an increase of the resonance value of $\omega_{B}$ (up to $\omega$ ). However, the analysis was restricted to multipactor resonance. Therefore the ECR effect was again overlooked because of the violation of the second equality in (17) at $\omega_{B}=\omega$. The authors of [13] also made an experimental investigation of the multipactor by varying the angle $\theta_{B}$ from 0 up to 45 degrees, however, without observing the ECR effect. Nevertheless, the ECR effect is possible even in the case of the single-surface multipactor on metal surfaces, as will be shown below.

As follows from the above analysis (see also [19, 20]), a strong ECR effect on the multipactor threshold can be expected only in the case when the electron flight time significantly exceeds the microwave period. Under typical multipactor conditions, the dc electric field pushes the secondary electrons back to the surface of emission. A strong magnetic field also causes fast electrons to return to the surface of emission when the tilt angle, $\theta_{B}$, is less than 30 degrees [13]. On the other hand, a significant ECR effect is possible when the microwave electric field has a nonzero component perpendicular to the permanent magnetic field (the value of $V_{\perp \omega}$ should be not too small compared to $V_{\omega}$ ). Therefore a strong ECR effect can be expected when the tilt angle, $\theta_{B}$, is around 45 degrees and the dc electric field is relatively weak and returns secondary electrons back in a time much longer than the microwave period.

\section{Numerical simulations of the low multipactor threshold on metal surfaces under the ECR condition}

The possibility of extremely low multipactor thresholds on a single metal surface was confirmed in numerical simulations using a simplified model. Within this model all microwave and dc fields are taken to be spatially uniform. Both microwave and dc electric fields are directed perpendicularly to the plane metal surface whereas the direction of the 
permanent magnetic field was varied. The software is based on the PIC algorithm [26], but the space charge effects were excluded from consideration which means that only the initial multipactor stage was simulated.

The seed electrons are launched from the metal surface $(x=0)$ uniformly during the first microwave period. The seed electrons have stochastic initial velocities governed by the Maxwell probability distribution. The motion of the electrons is governed by equations (1) and each collision of an electron with the metal surface is accompanied by secondary electron emission which is considered as a stochastic process. Its probability distribution is governed by electron impact energy and chosen so as to correspond to Vaughan's approximation [27] for the average value, $\sigma\left(W_{i m p}\right)$, of the secondary emission yield:

$$
\begin{gathered}
\sigma \equiv 0 \text { for } w<0, \\
\sigma=\sigma_{\max } \cdot[w \exp (1-w)]^{s}, s=0.62 \text { for } 0<w<1, s=0.25 \text { for } w \geq 1,
\end{gathered}
$$

where $\sigma_{\max }=\sigma_{m} \cdot\left(1+\phi^{2} / 4 \pi\right)$ denotes the maximum value of the secondary emission yield including its dependence on electron impact angle, $\phi$, (with respect to the normal to the surface $), \quad w=\left(W_{i m p}-W_{\min }\right) /\left(W_{\max }-W_{\min }\right), W_{\text {imp }}$ denotes the electron impact energy, $W_{\max }=W_{m} \cdot\left(1+\phi^{2} / 2 \pi\right)$, and the parameters $\sigma_{\max }, W_{\max }, W_{\min }$ are determined by material and surface treatment of the metal plate. The secondary electrons are assumed to start with stochastic initial velocity governed by the same Maxwellian probability distribution as the seed electrons. They also move under the action of the microwave field until their collisions with the wall which is accompanied by a release of new secondary electrons and the process is repeated as long as required.

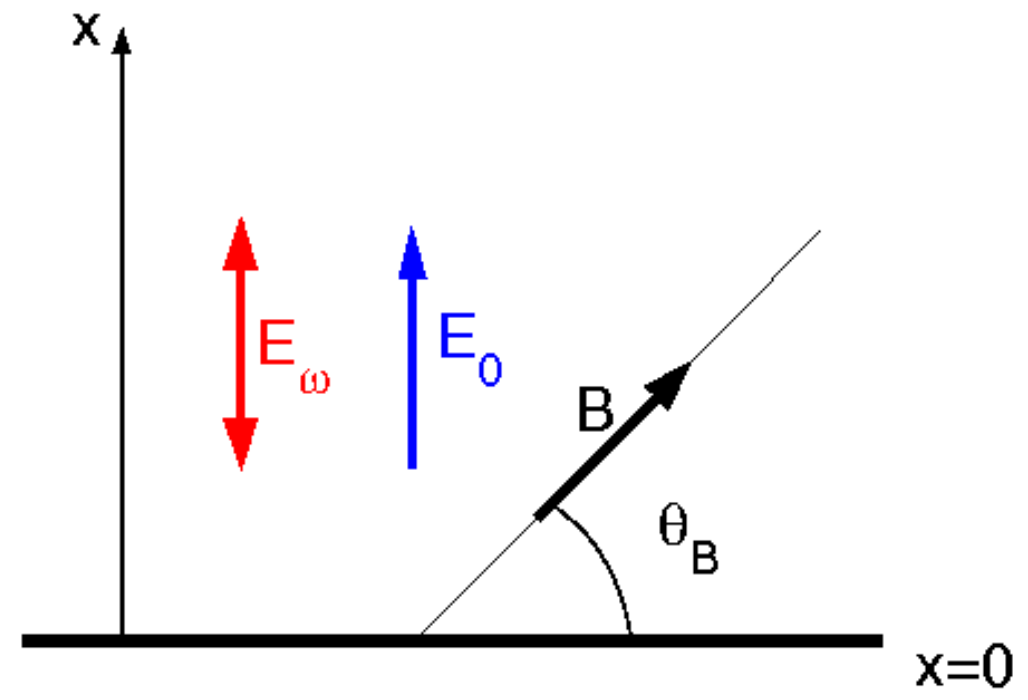


Fig. 3. The simulation geometry. The thick black line represents the emission surface, $x=0$. The red and blue arrows represents the rf and dc electric fields, which are normal to the surface, whereas the black arrow representing the magnetic field is tilted an angle, $\theta_{B}$, with respect to the surface.

The simulations were carried out using the following parameters for the secondary emission: $\sigma_{m}=2.25, W_{\min }=-8 \mathrm{eV}, W_{m}=333 \mathrm{eV}$ which corresponds to copper (the first cross over point corresponds to the impact energy $W_{1}=37 \mathrm{eV}$ ) as used in previous studies $[28,29]$. The field frequency was taken to be $30 \mathrm{GHz}$. Three simulation series were completed using a fixed value $\left(E_{0}=0, E_{0}=1.4 \mathrm{kV} / \mathrm{cm}, E_{0}=3 \mathrm{kV} / \mathrm{cm}\right)$ for the dc electric field in each series. In each series, the tilt angle, $\theta_{B}$, was varied from zero up to 90 degrees (see Fig. 3 for the simulation geometry), the value of $\omega_{B} / \omega$ varied within the range $0.4<\omega_{B} / \omega<1.2$ and the amplitude of the microwave field varied from $5 \mathrm{kV} / \mathrm{cm}$ up to 19 $\mathrm{kV} / \mathrm{cm}$. For each set of parameters, the simulations were started with $N_{0}=10^{3}$ seed electrons and stopped after 100 microwave periods. The multipactor threshold was determined as the lowest microwave amplitude at which the final electron number exceeded the number of seed electrons. The simulation results are presented in Figs. 4-6 where the dependence of the multipactor threshold on strength and orientation of the magnetic field is shown for different values of the dc electric field.

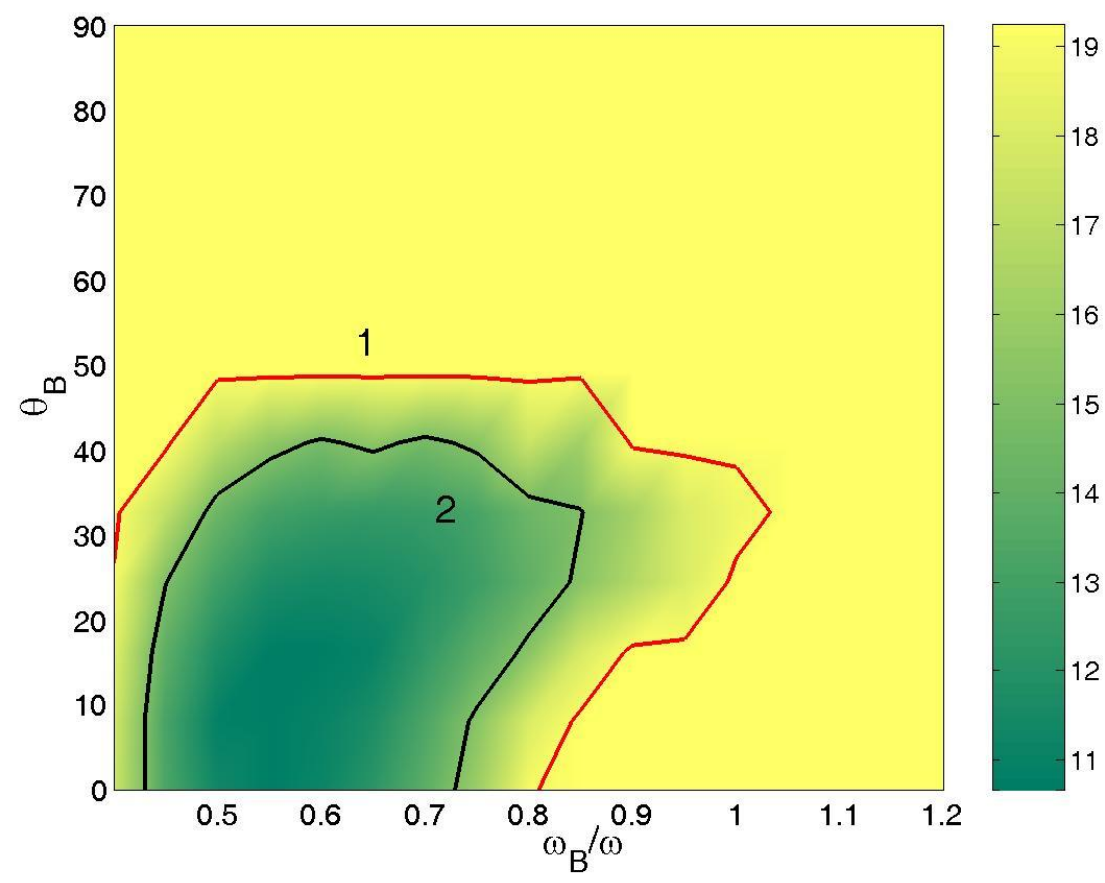


Fig. 4. The threshold amplitude (shown by color map in $\mathrm{kV} / \mathrm{cm}$ ) of the microwave field for the single-surface multipactor on a metal surface. The figure shows its dependence on the ratio $\omega_{B} / \omega$ and tilt angle $\theta_{B}$ between the permanent magnetic field and the metal surface. The calculations were completed with zero dc electric field. The contour line 1 corresponds to the amplitude, $E_{\omega}=19 \mathrm{kV} / \mathrm{cm}$ (in the parameter region outside this line, the multipactor avalanche does not grow within the simulation time). The contour line 2 corresponds to the amplitude $E_{\omega}=15 \mathrm{kV} / \mathrm{cm}$.

The multipactor region (surrounded by the contour line 1 in Fig. 4) looks very similar to that found in [13] as the region of the single-surface resonance of the first order. Inside this region, the multipactor threshold is close to that determined by the equality $V_{\perp \max }=V_{0}+2 V_{\perp \omega}=V_{1}$ (cf. (6) and (16)). In this case, the threshold minimum is about 11 $\mathrm{kV} / \mathrm{cm}$ and it is attained when the permanent magnetic field is parallel to the metal surface. On the other hand, Fig. 4 clearly demonstrates that in the case of zero dc electric field, the multipactor threshold is considerably higher under the ECR condition, which means that ECR itself is not favorable for the multipactor to occur.

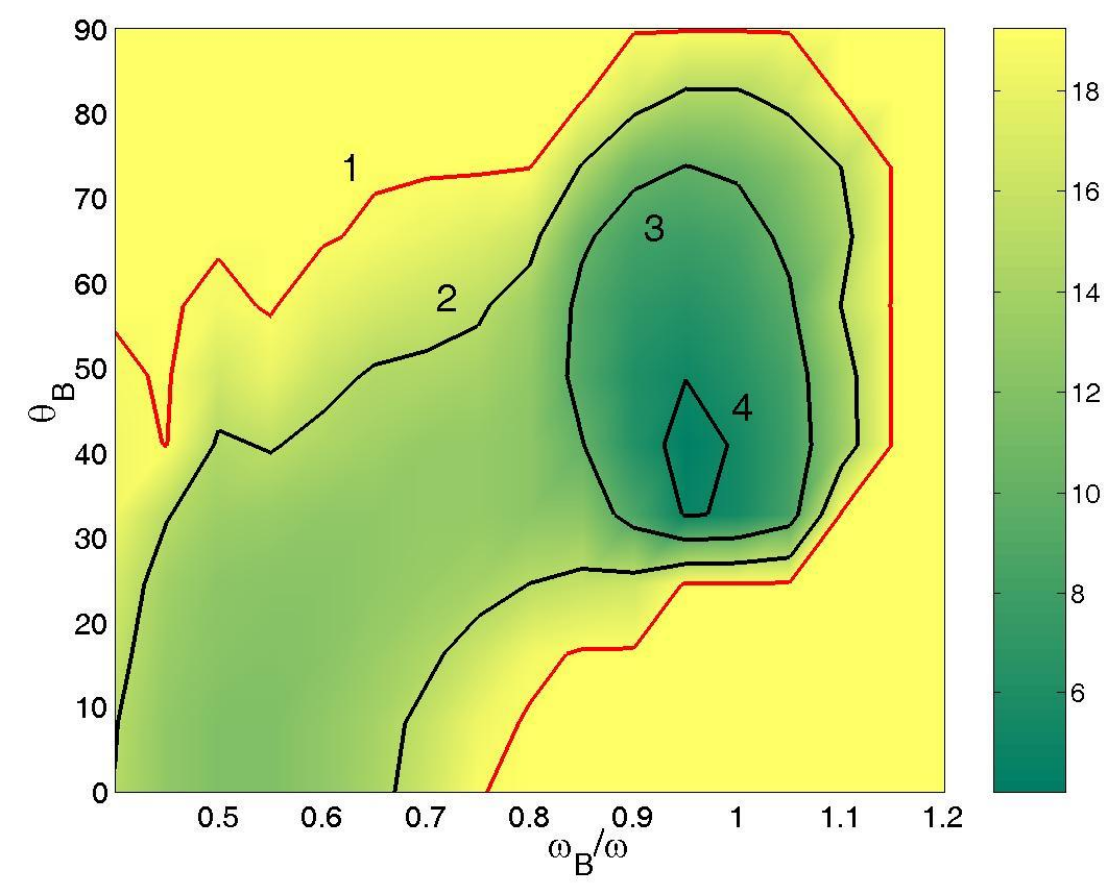

Fig. 5. The same as in Fig. 4 but the calculations are completed with a dc electric field $E_{0}=1.4 \mathrm{kV} / \mathrm{cm}$. The contour line 1 corresponds to the amplitude, $E_{\omega}=19 \mathrm{kV} / \mathrm{cm}$ (in the parameter region outside this line the multipactor avalanche does not grow within the simulation time). The contour lines 2,3 , and 4 correspond to the amplitudes 15,10 , and 5 $\mathrm{kV} / \mathrm{cm}$ respectively. 
Fig. 5 shows that the action of a weak dc electric field significantly affects the situation. In this case, the ECR effect results in a considerable reduction of the multipactor threshold and this reduction is most pronounced for tilt angles between 30 and 40 degrees, which is close to the theoretical predictions. In complete agreement with the theory, an increase in the dc electric field is accompanied by a weakening of the ECR effect, as demonstrated in Fig. 6 .

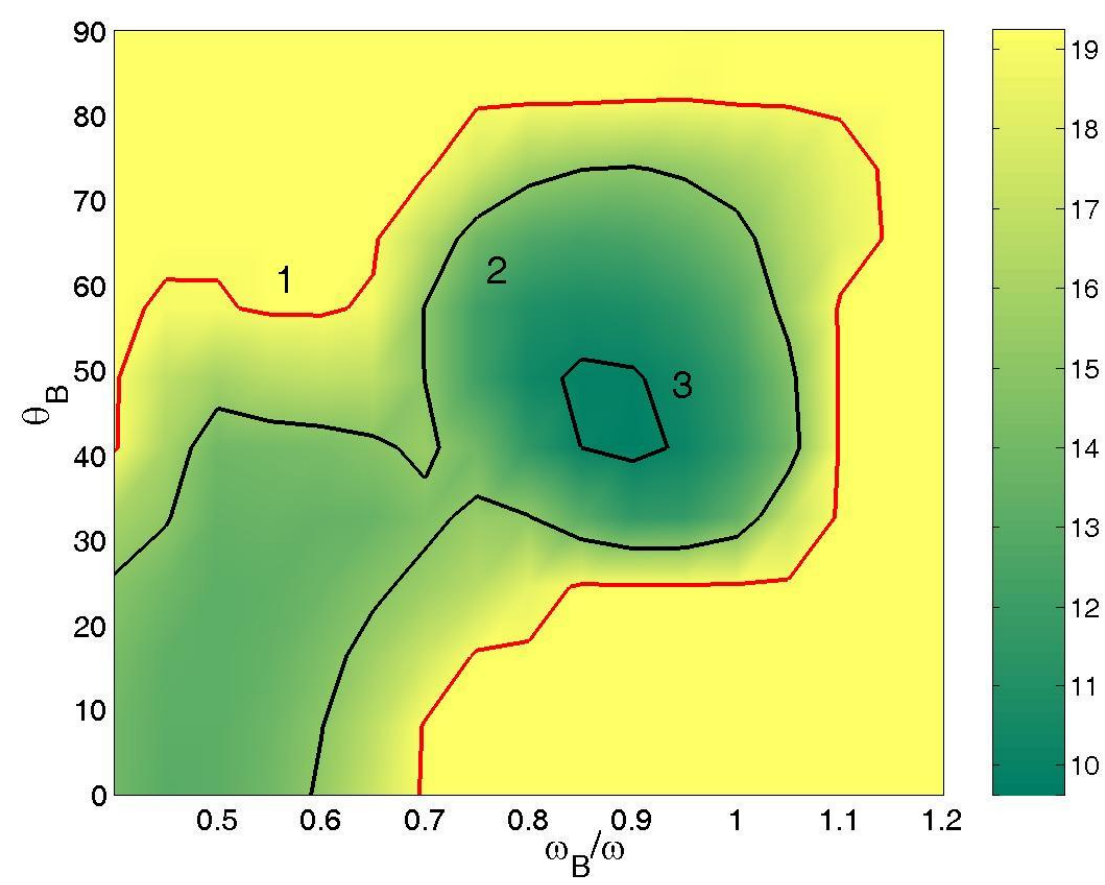

Fig. 6. The same as in Fig. 4, but the calculations are completed with a dc electric field $E_{0}=3 \mathrm{kV} / \mathrm{cm}$. The contour line 1 corresponds to the amplitude, $E_{\omega}=19 \mathrm{kV} / \mathrm{cm}$ (in the parameter region outside this line the multipactor avalanche does not grow within the simulation time). The contour lines 2 and 3 correspond to the amplitude 15 and $10 \mathrm{kV} / \mathrm{cm}$ respectively.

\section{Experimental indication of the low multipactor threshold on metal surfaces under the ECR condition}

The above conclusion on the possibility of extremely low multipactor thresholds on metal surfaces under the ECR condition was unexpectedly confirmed in a testing campaign of high power gyro-klystrons designed at the Institute of Applied Physics for the International program of particle acceleration. Within the first test campaign, the device prototype was fed by an electron beam with the energy $350 \mathrm{keV}$ and a current up to 120 A. According to 
engineering calculations, the output was expected to be an electromagnetic pulse with frequency $30 \mathrm{GHz}$, peak power about $15 \mathrm{MW}$, and duration of about 1 microsecond. However, all attempts to obtain such a pulse failed. Using a beam with lower current $70 \mathrm{~A}$, it became possible to obtain a pulse with the expected duration, but with a smaller power of 7 MW. However, an increase in current was not accompanied by a corresponding increase in the output power. The expected high power was achieved only in pulses with considerably shorter duration (not longer than 300 nanoseconds). The experimental results made it possible to assume that microwave power was absorbed in the device output section. To clarify the situation after several thousands shots, the output section was cut and a number of melting traces were found on its inner surface (see Fig. 7).

a

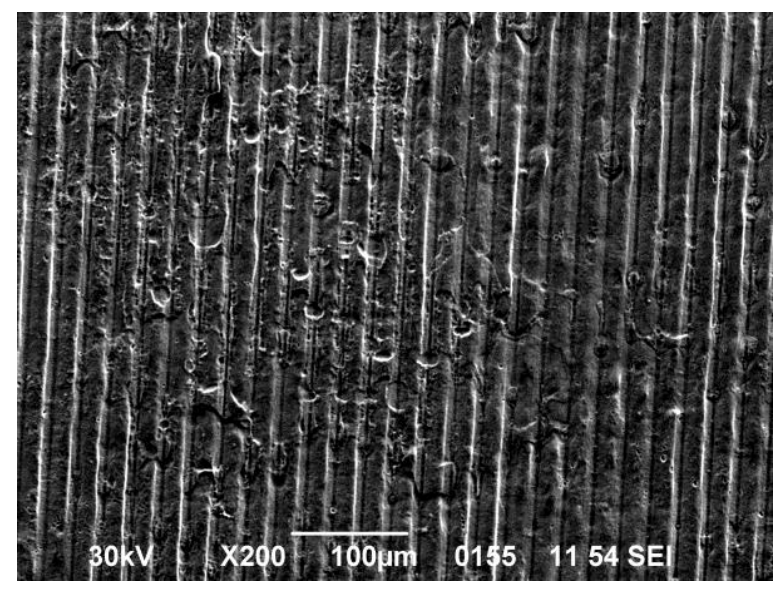

b

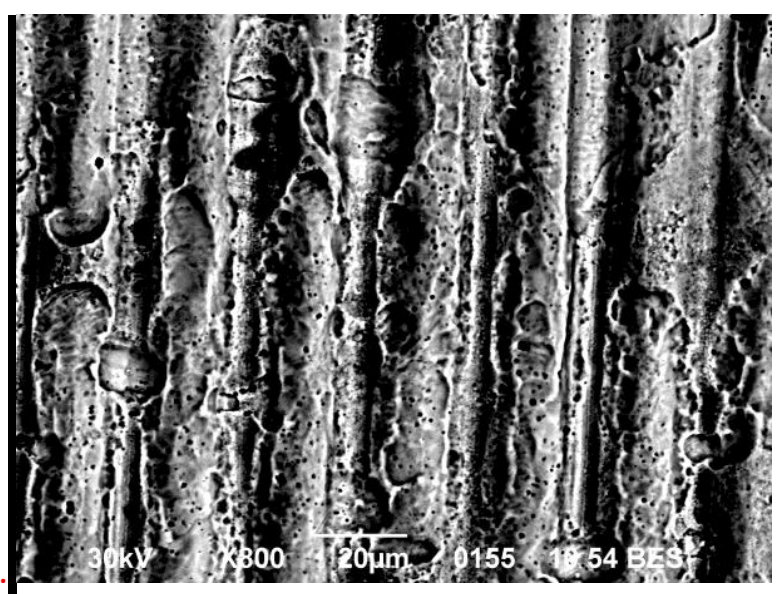

Fig. 7. Electron microscope images of then inner surface of the gyro-klystron output section at different magnification. The regular texture was introduced during fabrication. The traces of melting appeared as a result of the device operation.

The output section of the gyro-klystron represents a circular waveguide, $7 \mathrm{~cm}$ in diameter and $80 \mathrm{~cm}$ in length, made of copper. During the device operation, the high power microwave radiation was transmitted through this waveguide as a running wave at mode $\mathrm{TE}_{53}$ with circular polarization. Along the waveguide axis, the electron beam was also transported (Fig. 8) after its work in the device cavities. This beam induces a dc electric field which strength is proportional to the beam current and is estimated to be $1.5 \mathrm{kV} / \mathrm{cm}$ when the beam current equals $100 \mathrm{~A}$. At the power $10 \mathrm{MW}$, the amplitude of the microwave field on the waveguide wall was about $E_{\omega}=8 \mathrm{kV} / \mathrm{cm}$, which is well below the conventional threshold for the single-surface multipactor on a copper surface. The microwave field in the last cavity (Q- 
factor is about 300) of the gyro-klystron was considerably stronger. However, no erosion traces were found on the cavity walls. It should also be noted that the parameters of the microwave field were constant along the output waveguide and so were also the parameters of the dc electric field induced by the electron beam. Nevertheless, the melting spots were localized only within a relatively narrow ring zone perpendicular to the waveguide axis. The width of this zone was about $1-2 \mathrm{~cm}$. The distinct localization of this zone can be caused only by the residual magnetic field which was created by superconducting coils in the last cavity of the gyro-klystron and which penetrated into the output waveguide. Inside the last cavity and in the beginning of the waveguide, the value of the magnetic induction field was close to 1.7 $\mathrm{T}$ which corresponds to ECR conditions for relativistic electrons having an energy of 350 $\mathrm{keV}$. Further along the waveguide, the magnetic field decreases and becomes negligible close to the waveguide end. Measurements of the residual magnetic field showed that the melting spots are localized in the zone where the ECR conditions are fulfilled for non-relativistic electrons which can contribute to the multipactor avalanche. The residual magnetic field has both parallel and normal components with respect to the waveguide walls. Therefore, taking into account the dc electric field induced by the electron beam, it seems possible to conclude that the conditions for a considerable reduction of the multipactor threshold could be fulfilled in the observed melting zone.

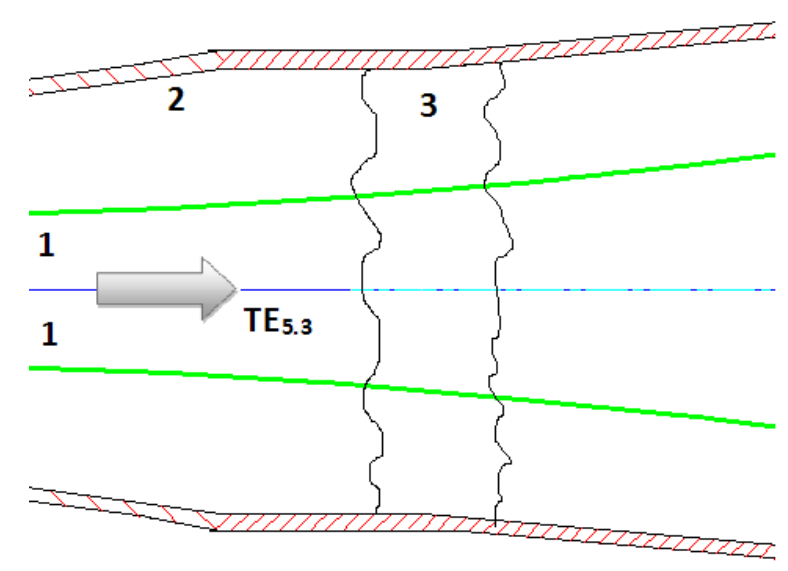

Fig. 8. Scheme of the output waveguide. Lines 1 illustrate the electron beam which has a tube-like shape, 2 is the waveguide wall (not in scale), lines 3 illustrate the position of the ECR region (not in scale) where $\omega_{B} \approx \omega$. 


\section{Discussion}

According to numerical simulations under ECR conditions, the rate of the electron avalanche is so high that a time of only a few nanoseconds is quite enough to achieve multipactor saturation. Consequently the pure multipactor hypothesis cannot explain the observed limitation of the high-power pulse duration at the level 300 ns. However, as was stated previously (se e.g. [1,2]), the multipactor itself does not absorb much microwave power and therefore it cannot directly stop the generation of high power microwaves. More probable is that the time of $300 \mathrm{~ns}$ is required for desorption of gaseous molecules, a process that is stimulated by multipactor on the waveguide walls [1].

According to the above analysis, ECR reduction of the multipactor threshold on metal surfaces takes place when the tilt angle between the magnetic field and the metal surface exceeds 30 degrees. It should be underlined that in the ECR region even local excess of the tilt angle over 30 degrees can result in a considerable reduction of the multipactor threshold if the size of the region with proper tilt angle is larger than the amplitude, $a_{\omega}=V_{\omega} / \omega$, of the electron oscillations in the microwave field. At the frequency $30 \mathrm{GHz}$ and the microwave field amplitude $E_{\omega}=10 \mathrm{kV} / \mathrm{cm}$, the amplitude of the electron oscillations is less than 10 micrometers. This means that even small-scale textures of the metal surface (which can be seen in Fig. 7) can be important for the multipactor threshold reduction. This is the case in the output waveguide of the experiment described above, where the small scale texture introduced by the fabrication process forms trenches and ridges with a typical size of around $20 \mu \mathrm{m}$. This leads to a situation where the average tilt angle of the magnetic field is actually below the critical 30 degrees, but the local tilt due to the surface texture varies, and even exceeds 45 degrees locally.

\section{Conclusion}

Single-surface multipactor can grow on metal surfaces at very low amplitudes of the microwave field, if the latter is superimposed on a permanent magnetic field which is strong enough to satisfy the condition of electron cyclotron resonance, i.e. $\omega_{B}=\omega$. The ECR effect on the multipactor threshold occurs if (and only if) there is a significant angle between the metal surface and the magnetic field line. In that sense, the most dangerous case is a tilt angle between 30 and 40 degrees. Another necessary prerequisite for the ECR multipactor effect is the presence of a relatively weak dc electric field which returns secondary electrons back to 
the surface of emission. The weaker the dc electric field, the stronger can the reduction of the multipactor threshold be, but the more sensitive is the effect to perturbations of the exact cyclotron resonance. 


\section{$\underline{\text { References }}$}

1. N.F. Kovalev, V.E. Nechaev, M.I. Petelin, N.I. Zaitsev, Scenario for output pulse shortening in microwave generators driven by relativistic electron beams. IEEE Trans. Plasma Sci. vol. PS-36, No. 3, pp. 246-251, (1998).

2. J.G. Power, W. Gai, S. H. Gold, A. K. Kinkead, R. Konecny, C. Jing, W. Liu, and Z. Yusof. Observation of Multipactor in an Alumina-Based Dielectric-Loaded Accelerating Structure. Phys. Rev. Letters. V. 92, No. 16, 164801 (pp.1-4) (2004).

3. Ming Yu. Power-Handling Capability for rf Filters, IEEE Microwave Magazine, v. 8, issue 5, pp. 88-97, (2007).

4. C.Chang, G.Z. Liu, H.J. Huang, C.H. Chen, and J.Y. Fang. Suppressing high-power microwave dielectric multipactor by sawtooth surface. Physics of Plasmas, v. 16, 083501 pp. 1-6 (2009).

5. R.L. Geng, H.S. Padamsee, Exploring multipacting characteristics of a rectangular waveguide. Proc. 1999 Particle Accelerator Conference, New York, (IEEE, Piscataway,1999), pp. 429-431.

6. R.L. Geng, H. Padamsee, S. Belomestnykh, P. Goudket, D.M. Dykes, R.G. Carter, Suppression of multipacting in rectangular coupler waveguides, Nuclear Instruments and Methods in Physics, Research A 508, 227-238 (2003).

7. P. Goudket, D.M. Dykes, R.L. Geng, S. Belomestnykh, H. Padamsee, R.G. Carter. STUDIES OF ELECTRON MULTIPACTING IN CESR TYPE RECTANGULAR WAVEGUIDE COUPLERS. Proceedings of EPAC 2004, Lucerne, Switzerland, pp.10571059 (2004).

8. C. Chang, G. Z. Liu, C. X. Tang, C. H. Chen, H. Shao, and W. H. Huang. Suppression of high-power microwave dielectric multipactor by resonant magnetic field. APPLIED PHYSICS LETTERS, v. 96, (2010), 111502 (3p).

9. Chao Chang, John Verboncoeur, Sami Tantawi, and Chunguang Jing. The effects of magnetic field on single-surface resonant multipactor. J. Appl. Phys. 110, 063304, pp. 1-5 (2011)

10. D. Gonzalez-Iglesias, A. M. Perez, S. Anza, J. Vague, B. Gimeno, V. E. Boria, D. Raboso, C. Vicente, J. Gil, F. Caspers, L. Conde. Multipactor in a Coaxial Line with the Presence of an Axial DC Magnetic Field. IEEE Electron Device Letters, vol. 33, no. 5, pp. 727-729, May 2012. 
11. Gaddy O.L., Holshauser D.F. A microwave frequency dynamic cross-field photomultiplier. IEEE. V. 51, No. 1, pp.153-162 (1963).

12. L. G. Blyakhman and V. E. Nechaev, Conditions for the onset of a vacuum resonant microwave discharge in a magnetostatic field. Sov. Phys. Tech. Phys.vol. 25, No. 4, pp. 430433 (1980) Translated from Zh. Tekh. Fiz ., 50, No 4, pp. $720-727$ (1980).

13. K. Sakamoto, T. Imai, Y. Ikeda, M. Saigusa, J. Sagawa, and T. Nagashima. Unipole Multipactoring Discharge in the LHRF Launcher. IEEE Transactions on Plasma Science, vol. PS-14, No. 4, pp. 548-553 (1986)

14. S. Riyopoulos, D. Chernin, D. Dialetis, Theory of Electron Multipactor in Crossed Fields. Phys, Plasmas, v. 2, No. 8, pp. 3194-3213, (1995).

15. M. Buyanova, V. Nechaev, E. Rakova, V. Semenov, N. Zharova, D. Anderson, M. Lisak, J. Puech, and L. Lapierre. Multi-phase regimes of Multipactor Breakdown, Proceedings of MULCOPIM 2005 (12-14 September 2005, ESA/ESTEC, Noordwijk, the Netherlands) pp. 135-142 (2005).

16. E.V. Ilyakov, I. S.Kulagin, and V. E.Nechaev. Experimental Study of a Saturated Multipactor in Crossed Fields. Radiophysics and Quantum Electronics, Vol. 52, No. 12, pp. 885-891 (2009).

17. D. H. Preist and R. C. Talcott. Qn the Heating of Output Windows of Microwave Tubes bv Electron Bombardment. IRE Transactions on Electron Devices, v. 8, issue 4, pp. 243-251 (1961).

18. J. R. M. Vaughan, Some high power window failures. IRE Trans. Electron Devices 8, pp. 302-308 (1961)

19. A. Valfells, L. K. Ang, Y. Y. Lau, and R. M. Gilgenbach. Effects of an external magnetic field, and of oblique radio-frequency electric fields on multipactor discharge on a dielectric. PHYSICS OF PLASMAS VOLUME 7, NUMBER 2, pp. $750-757$ (2000)

20. N. K. Vdovicheva, A.G. Sazontov, and V. A. Sazontov. Influence of an External Magnetic Field on the Threshold of Multipactor Onset on a Dielectric Surface. Radiophysics and Quantum Electronics, Vol. 50, No. 2, pp. 108-122 (2007)

21. W.J. Schrader A new mode of cyclotron resonance multipacting, Physica, v 40, n 2 , pp. 223-228, (1968).

22. A.J.Hatch and B.Williams. Multipacting modes of high-frequency gaseous breakdown. Phys. Rev., v. 112, No. 3, pp. 681-685, (1958). 
23. A. Kryazhev, M. Buyanova, V. Semenov, D. Anderson, M. Lisak, J. Puech, L. Lapierre, and J. Sombrin. Hybrid resonant modes of two-sided multipactor and transition to the polyphase regime, Phys. Plasmas, v. 9, No. 11, pp. 4736-4743 (2002)

24. E. F. Vance, One-sided multipactor discharge modes. J. Appl. Phys., v. 34, No. 11. pp.3237-3242, (1963).

25. A. V. Gaponov, and M. A. Miller, Potential Wells for Charged Particles in a HighFrequency Electromagnetic Field, Sov. Phys. JETP, v. 7, pp. 168-169 (1958).

26. J.P. Verboncoeur, A.B. Langdon, and N. T. Gladd, An Object-Oriented Electromagnetic PIC Code, Computer Physics Communications, Vol. 87, Nos. 1-2, pp. 199211, (1995).

27. J. R. M. Vaughan, Secondary Emission Formulas. IEEE Trans. Electron Devices, v. 40, No. 4, pp. 830-830 (1993).

28. V.E. Semenov, N.A. Zharova, R. Udiljak, D. Anderson, M. Lisak, J. Puech. Multipactor in a coaxial transmission line. II. Particle-in-cell simulations. PHYSICS OF PLASMAS 14, 033509 (pp. 1-7) (2007).

29. V.E.Semenov, N.A.Zharova, D.Anderson, M.Lisak, and J.Puech. Simulations of Multipactor in Circular Waveguides. Physics of Plasmas, Vol. 17, 123503, 7pp. (2010). 